\title{
Ethnobotanical knowledge and folk medicinal significance of the flora of district Gujrat, Punjab, Pakistan
}

\author{
SYED SAQIB ALI, KHALID HUSSAIN, KHALID NAWAZ, KHIZAR HAYAT BHATTI, ZOBIA \\ BASHIR, ARIFA NAZEER, USMAN ARIF, SANA JAFAR, EJAZ HUSSAIN SIDDIQI
}

University of Gujrat

Gujrat

Pakistan

*corresponding author: e-mail: khalid.hussain@uog.edu.pk

\section{Summary}

Introduction: Medicinal plants are the natural source of medicines used in treatment of many diseases among local communities of various countries. It is also the raw material for the pharmaceutical industry. Pakistan is a country with a variety of medicinal plants, comprehensively studied in some areas, while others still need more studies of their flora.

Objectives: The study was performed to catalogue the flora of district Gujrat, Punjab, Pakistan and to assess its ethnomedicinal importance.

Methods: A field survey was conducted in the Gujrat district (including Sarai Alamgir, Gujrat and Kharian) in 2019-2020 to collect the flora. Nearly 200 people of various age groups were interviewed and the questionnaire was filled, the gender and professions of the participants were also noted.

Results: The plant species collected belonged to 32 different Angiosperm families i.e. Poaceae, Brassicaceae, Fabaceae, Asteraceae, Moraceae, Amaranthaceae, Apiaceae, Myrtaceae, Rutaceae, Solanaceae, Malvaceae, Euphorbiaceae and Lamiaceae. The obtained material consisted of 30 herbs, 20 shrubs and 22 trees. The floral parts used most often were leaves (63\%) followed by: flower (41\%), fruit (28\%), stem (16\%), seed (16\%), bark (14\%), root (13\%), whole plant (12\%), underground part (3\%) and latex (3\%). According to the study, the highest RFC (Relative Frequency of Citation) values were 0.1 in Cassia fistula L. (0.25) and the smallest RFC values were observed in Stellaria media (L.) Vill. - 0.025. The highest UV (Use Values) were noted for Achyranthes aspera L. at 0.9. In disease category, the highest value (0.67) of ICF (Informant Consensus Factor) was observed for stomach and skin infections, while the lowest ICF value was noted for diabetes and gonorrhoea (0.27).

Conclusions: It was concluded that the Gujrat district is rich in useful plants that can be used in the preparation of various medicines as well as be sold on the herbal market. 


\section{Keywords: District Gujrat, medicine, ethnomedicinal flora, survey, native people \\ Słowa kluczowe: prowincja Gujrat, lek, rośliny lecznicze używane w medycynie ludowej, badanie ankietowe, rdzenni mieszkańcy}

\section{INTRODUCTION}

Gujrat is a district of Pakistan, situated between the Jhelum and Chenab rivers. It is spread over an area of $3,192 \mathrm{~km}^{2}$ and consists of three tehsils (townships): Sarai Alamgir, Kharian and Gujrat. It has a moderate climate but the temperatures vary significantly between seasons, with very warm summers and cold winters. During summer, temperatures rise up to $46^{\circ} \mathrm{C}$, though only for a relatively short period due to the nearby hills of Azad Jammu and Kashmir. During winter temperatures drop below $2^{\circ} \mathrm{C}$ [1].

In Pakistan, there is a significant diversity of climate, geographical areas and conservation areas [2]. They contain a variety of large, speckled and various other kinds of plants. Almost 600 species of angiosperm flora are recorded in Kashmir and Pakistan [3]. It has been concluded in the study of ethnomedicinal flora, that these plants were utilized by the initial inhabitants of these areas [4]. In developing countries, almost $80 \%$ of people are utilizing traditional ethnomedicinal flora as cures for various ailments [5]. There are a number of plant species exhibiting pharmaceutical properties growing in the wild, making it easy for people to gather and utilize them [6]. By utilization of infective microorganisms, the flora and fauna act against bacteria and fungi by use of chemicals extraction from important deep enact flora [7].

There are approximately 6000 species which are gathered and used as cures for various diseases and 600 species are used as pharmaceutical sources [8]. For particular health disorders, the families of flora such as Apiaceae, Fabaceae, Poaceae, Solanaceae and Moraceae are consumed. They are involved in various cellular activities like radical scavenging, stimulant, alimentary canal, portliness diseases caused by microorganisms, inflammation, pain killers. They are also a good source for collection of bio pesticides [9]. According to latest Pharmacopoeia, approximately $25 \%$ of drugs are described due to its importance in medicinal plants. The chemicals that are obtained from pharmaceutical flora are almost $25 \%$ in their number. Pharmaceutical flora is also consumed by the native people of Gujrat to treat various ailments [10].
This study was undertaken to explore the flora in district Gujrat, Punjab, Pakistan and its medicinal importance through folk knowledge.

\section{MATERIALS AND METHODS}

Field surveys were conducted in three tehsils of district Gujrat: Sarai Alamgir, Gujrat and Kharian in 2019 and 2020, to collect the flora. Map of the selected area is given in figure 1. A number of field tours were conducted. Approximately 200 people of different age groups, genders and professions were interviewed and filled a questionnaire for the documentation. Plants collected during the survey were dried and preserved in herbarium sheets. Plants were identified with the help of "Flora of Pakistan".

\section{Data analysis}

The data was analysed for different parameters to find the ethnomedicinal importance of the flora.

\section{Use value (UV)}

Use Value (UV) was used to determine the relative importance of a given species collected from the area. It was calculated by the following formula:

$\mathrm{UV}=\Sigma \mathrm{U} / \mathrm{N}$, where:

$\mathrm{U}$ - the number of citations per specific plant,

$\mathrm{N}$ - the number of informants.

\section{Informant consensus factor (ICF)}

Information of related homogeneity mixture of disease category obtained from informants was calculated by this formula:

$\mathrm{ICF}=(\mathrm{Nur}-\mathrm{Nt}) /(\mathrm{Nur}-1)$, where:

Nur - the number of citations from informants 


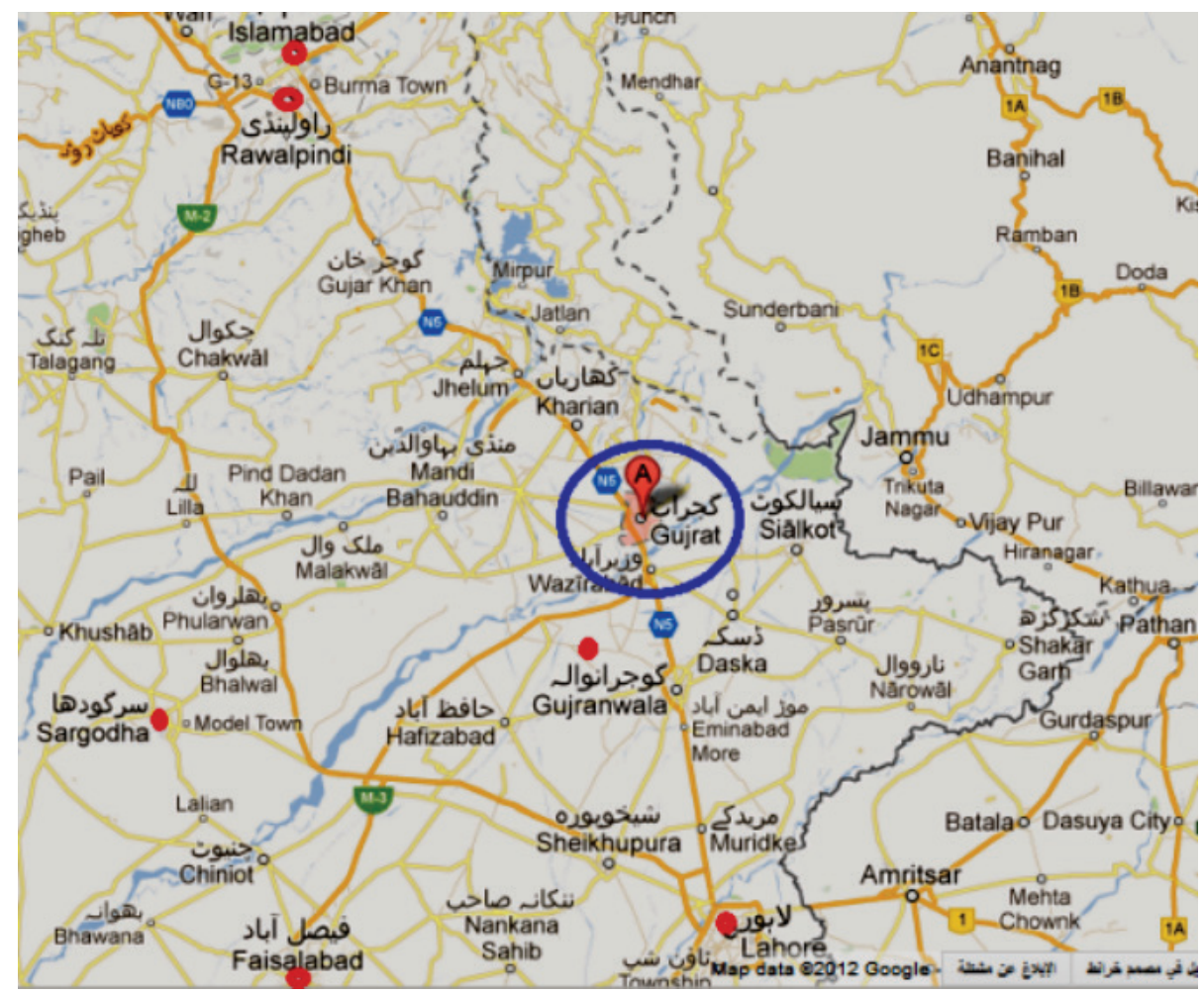

Figure 1

Map of study area (district Gujrat, Punjab, Pakistan)

for a particular plant-use category,

$\mathrm{Nt}$-the number of species or taxa utilized by all the informants for that specific plant use category.

ICF had ranged from 0 to 1 , where ICF value of 1 indicated the highest level of informant consensus and 0 was the lowest value.

\section{Fidelity level (FL)}

The fidelity level was calculated to search out the value of the species related to medicines:

$\mathrm{FL}(\%)=(\mathrm{Np} / \mathrm{N}) \times 100$,

where:

$\mathrm{Np}$ - the number of species that is present in a specific category.

For accurate sum consumption for particular species, $\mathrm{N}$ was used.

\section{Relative frequency of citation (RFC)}

RFC was calculated by the given formula:

$\mathrm{RCF}=\mathrm{FC} / \mathrm{N}$,

where:
FC - the number of informants using that species,

$\mathrm{N}$ - the total number of informants in the survey.

Ethical approval: The conducted research is not related to either human or animal use.

\section{RESULTS}

\section{Distribution of families}

During the surveys, it was found that plant species collected belong to 32 angiosperm families. Ranking of families was based on the number of species present in the area, i.e. Poaceae was placed at $1^{\text {st }}$ rank due to the presence of the highest number of species in the area which was 9 species. Brassicaceae and Fabaceae had 6 species each, and Asteraceae had 5 species in the study area.

There were 4 species for families Moraceae and Amaranthaceae. Apiaceae, Myrtaceae and Rutaceae had 3 species, Euphorbiaceae, Lamiaceae, Malvaceae, Solanaceae, Amaryllidaceae and Apocyanaceae had 2 plant species, while all the other identified families had only 1 plant species (fig. 2). 


\section{Plant identification and ethnomedicinal uses}

The data related to botanical name, family, local name, UV, RFC, local uses, part used, prescription, dosage, form of use and diseases is described in table 1. All these medicinal plants are traditionally used in healing, according to the informants. The most commonly collected plant at various locations was Acacia modesta which was used in healing wounds, once a day. Powder made from the bark of Achras sapota f. asperma was used during childbirth to control pain. Wounds and pimples were cured with the bulb of $\mathrm{Al}$ lium cepa, Aloe vera was helpful in removing dandruff from hair with other ingredients like yoghurt, egg and oil. Stem and leaves of Amaranthus viridis were mixed with rice and used to ease menstrual pain. Seeds of Bombax ceiba mixed with black musli and sufeed musli were recommended in diarrhoea, used once a day. Oil obtained from the seeds of Brassica campestris var. sarson. was helpful for colds, joints and arthritis. For general body pain, oil from mustard plant mixed with the leaves of Calotropis procera were rubbed on the body surface. Oil obtained from Carthamus oxycantha was the best for respiratory tract problems. Decoction made from the bark of Cassia fistula was utilized for constipation. People used leaves of the Chenopodium album for the pain of joints and kidney. For fever, lemon water was used as herbal tea with other spices. Herbal tea made from the leaves of Cymbopogon citratus was used in cholera and stomach problems. Leaves of Eucalyptus globulus were ground to make powder that was helpful in digestion. Poultice made from the leaves of Lepidium bonariense was used for body pain and diabetes. For digestive disorders, leaves of the Mentha arvensis was recommended thrice a day. For general body weakness, people used Phoenix dactylifera twice a day with milk. Leaves of the Stellaria media were directly applied on the surface of a wound for healing. The roots of Withania somnifera were used to control symptoms of stress. Fruit obtained from the Ziziphus jujuba was used for the treatment of stomach and hepatitis disorders (tab. 1).

According to this study, it can be concluded that use value (UV) was 0.23-0.9. Achyranthes aspera showed highest UV (0.6) whereas Spinacae oleraece had lowest UV (0.23) among all the species collected from the area (tab. 2). Relative frequency of citation (RFC) and UV were used to search floral species that can be utilized for the manufacturing of medicines. It has been observed that the flora of the area showed RFC value ranged from 0.025 to 0.1 (tab. 1). Floral species of low RFC value was Stellaria media (0.025) whereas the highest value was in Cassia fistula (0.25).

\section{Parts used in ethnomedicine}

The plants parts used in medicines were leaves, roots, stem, latex, seeds and whole plant (tab. 1). For the preparation of basic medications, floral species were used in the form of herbal tea, distillation, decoction and paste powder. Percentage of the used parts was as follows: leaves (63\%), flower (41\%), fruit (28\%), stem

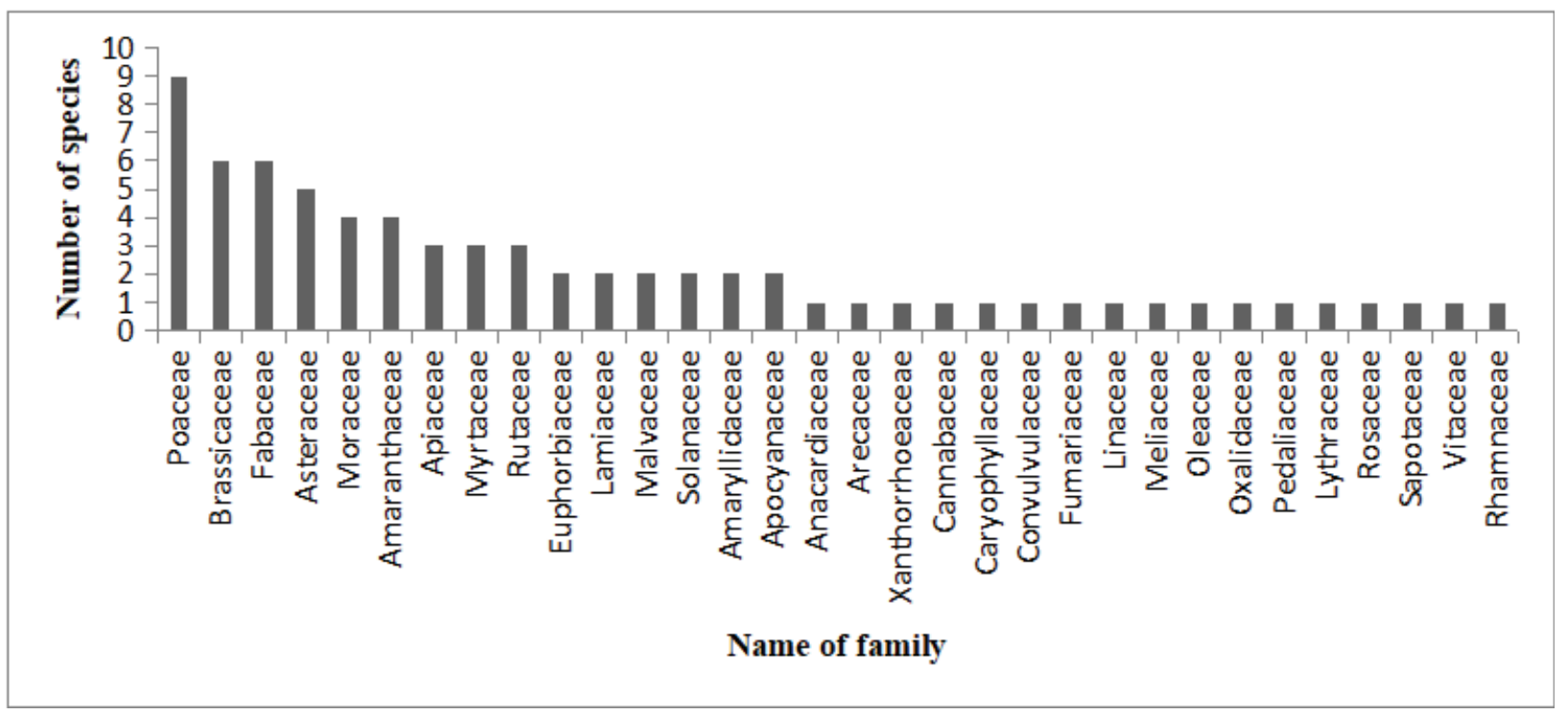

Figure 2

Family index with highest number of species used in study area 
Table 1

Identification of plants, medicinal values and methods of use

\begin{tabular}{|c|c|c|c|c|c|c|c|c|c|c|}
\hline $\begin{array}{l}\text { Botanical } \\
\text { name }\end{array}$ & Family & $\begin{array}{l}\text { Common } \\
\text { name }\end{array}$ & UV & RFC & Local use & Part use & Prescription & $\begin{array}{l}\text { Time of } \\
\text { use }\end{array}$ & $\begin{array}{l}\text { Form } \\
\text { of use }\end{array}$ & Use of drug \\
\hline $\begin{array}{l}\text { Acacia } \\
\text { modesta } \\
\text { Wall. }\end{array}$ & Fabaceae & $\begin{array}{l}\text { Phulai, jangli } \\
\text { kikar }\end{array}$ & 0.733 & 0.075 & $\begin{array}{l}\text { Teeth problems, } \\
\text { cure gastric } \\
\text { problems }\end{array}$ & $\begin{array}{l}\text { Bark and } \\
\text { stem }\end{array}$ & $\begin{array}{l}\text { Take bark } \\
\text { and gum of } \\
\text { this plant and } \\
\text { make solution }\end{array}$ & $\begin{array}{l}1 \text { time } \\
\text { per day }\end{array}$ & $\begin{array}{l}\text { Dry } \\
\text { and } \\
\text { also } \\
\text { fresh } \\
\text { form }\end{array}$ & Use for wound \\
\hline $\begin{array}{l}\text { Achras } \\
\text { sapota } \mathrm{f} . \\
\text { asperma M. } \\
\text { Gómez }\end{array}$ & Sapotaceae & Chiku & 0.33 & 0.045 & $\begin{array}{l}\text { Diuretic and } \\
\text { useful in fever. }\end{array}$ & $\begin{array}{l}\text { Bark, fruit, } \\
\text { seed }\end{array}$ & $\begin{array}{l}\text { Bark is use to } \\
\text { make powder } \\
\text { that is used } \\
\text { on the body } \\
\text { of female after } \\
\text { birth }\end{array}$ & $\begin{array}{l}1 \text { time } \\
\text { per day }\end{array}$ & $\begin{array}{l}\text { Dry } \\
\text { form }\end{array}$ & $\begin{array}{l}\text { For delivery } \\
\text { purpose in } \\
\text { female }\end{array}$ \\
\hline $\begin{array}{l}\text { Achyranthes } \\
\text { aspera } \mathrm{L}\end{array}$ & Amaranthaceae & Puth Kanda & 0.9 & 0.08 & $\begin{array}{l}\text { Cough, kidney } \\
\text { problem, } \\
\text { dysentery, } \\
\text { skin disease, } \\
\text { rheumatism, } \\
\text { stomach pain } \\
\text { and toothache }\end{array}$ & $\begin{array}{l}\text { Whole plant } \\
\text { body }\end{array}$ & $\begin{array}{l}\text { Puthkanda } \\
\text { leaves and } \\
\text { honey bee }\end{array}$ & $\begin{array}{l}3 \text { times } \\
\text { per day }\end{array}$ & $\begin{array}{l}\text { Dry } \\
\text { form }\end{array}$ & Cough \\
\hline $\begin{array}{l}\text { Albizia } \\
\text { lebbeck (L.) } \\
\text { Benth }\end{array}$ & Fabaceae & Shrin & 0.6 & 0.065 & $\begin{array}{l}\text { Lung problems, } \\
\text { blood } \\
\text { purification } \\
\text { abdominal } \\
\text { tumours, boils, } \\
\text { cough, hernia, } \\
\text { secondary } \\
\text { infertility, eye } \\
\text { infections, flu } \\
\text { and tonic }\end{array}$ & Stem, bark & $\begin{array}{l}\text { Leaves boil in } \\
\text { water }\end{array}$ & $\begin{array}{l}1 \text { time } \\
\text { per day }\end{array}$ & $\begin{array}{l}\text { Fresh } \\
\text { leaves }\end{array}$ & $\begin{array}{l}\text { Showering } \\
\text { baby after birth }\end{array}$ \\
\hline $\begin{array}{l}\text { Allium } \\
\text { cepa L. }\end{array}$ & Amaryllidaceae & Piyaz, Onion & 0.33 & 0.05 & $\begin{array}{l}\text { Diabetes, } \\
\text { blood sugar } \\
\text { level, diuretic, } \\
\text { arteriosclerosis }\end{array}$ & Bulb & $\begin{array}{l}\text { After boiling } \\
\text { the bulb of } \\
\text { onion use for } \\
\text { wound }\end{array}$ & $\begin{array}{l}1 \text { time } \\
\text { per day }\end{array}$ & $\begin{array}{l}\text { Fresh } \\
\text { form }\end{array}$ & $\begin{array}{l}\text { Use form } \\
\text { pimple } \\
\text { treatment }\end{array}$ \\
\hline $\begin{array}{l}\text { Allium } \\
\text { sativum L. }\end{array}$ & Amaryllidaceae & Garlic, Thom & 0.6 & 0.06 & $\begin{array}{l}\text { Liver disorder, } \\
\text { bronchitis, } \\
\text { fever, cancer } \\
\text { disease, low } \\
\text { blood pressure }\end{array}$ & $\begin{array}{l}\text { Leaves, } \\
\text { bulbils }\end{array}$ & $\begin{array}{l}\text { Use sap after } \\
\text { gridding the } \\
\text { lassan }\end{array}$ & $\begin{array}{l}2 \text { times } \\
\text { per day }\end{array}$ & $\begin{array}{l}\text { Dry } \\
\text { form }\end{array}$ & Eyes treatment \\
\hline $\begin{array}{l}\text { Aloe vera } \\
\text { (L.) Burm.f. }\end{array}$ & Xanthorrhoeaceae & Kawar gandal & 0.42 & 0.1 & $\begin{array}{l}\text { Skin disorders, } \\
\text { inflammation, } \\
\text { itchiness, } \\
\text { skin disorder, } \\
\text { stomach } \\
\text { problem and } \\
\text { gum bleeding }\end{array}$ & Leaves & $\begin{array}{l}\text { Yoghurt, egg, } \\
\text { Aloe vera } \\
\text { jel and oil } \\
\text { homogenize it }\end{array}$ & $\begin{array}{l}1 \text { time } \\
\text { per week }\end{array}$ & $\begin{array}{l}\text { Fresh } \\
\text { form }\end{array}$ & $\begin{array}{l}\text { Remove } \\
\text { dandruff }\end{array}$ \\
\hline $\begin{array}{l}\text { Amaranthus } \\
\text { viridis } \mathrm{L} .\end{array}$ & Amaranthaceae & $\begin{array}{l}\text { Green } \\
\text { amaranth, } \\
\text { Cholai }\end{array}$ & 0.33 & 0.055 & $\begin{array}{l}\text { Fever, asthma, } \\
\text { liver disorder, } \\
\text { urinary, eye } \\
\text { problem and } \\
\text { menstrual } \\
\text { problem in } \\
\text { female }\end{array}$ & $\begin{array}{l}\text { Leaves and } \\
\text { stem }\end{array}$ & $\begin{array}{l}\text { Stem and } \\
\text { leaves after } \\
\text { grinding are } \\
\text { mixed with the } \\
\text { rice water }\end{array}$ & $\begin{array}{l}1 \text { time } \\
\text { per day }\end{array}$ & $\begin{array}{l}\text { Fresh } \\
\text { form }\end{array}$ & $\begin{array}{l}\text { To control } \\
\text { menstruation }\end{array}$ \\
\hline $\begin{array}{l}\text { Avena } \\
\text { sativa } \mathrm{L} .\end{array}$ & Poaceae & Jai, Oat & 0.43 & 0.06 & $\begin{array}{l}\text { Constipation, } \\
\text { cure of tension } \\
\text { and skin } \\
\text { allergies }\end{array}$ & Seed & $\begin{array}{l}\text { Gridding seed, } \\
\text { sugar and } \\
\text { water }\end{array}$ & $\begin{array}{l}1 \text { time } \\
\text { per day }\end{array}$ & $\begin{array}{l}\text { Dry } \\
\text { form }\end{array}$ & $\begin{array}{l}\text { To control } \\
\text { temperature of } \\
\text { body }\end{array}$ \\
\hline
\end{tabular}




\begin{tabular}{|c|c|c|c|c|c|c|c|c|c|c|}
\hline $\begin{array}{l}\text { Bombax } \\
\text { ceiba L. }\end{array}$ & Malvaceae & Simbel & 0.7 & 0.08 & $\begin{array}{l}\text { Snake bites, } \\
\text { dysentery, } \\
\text { inflammation, } \\
\text { blood } \\
\text { purification, } \\
\text { genital organs } \\
\text { treatment, } \\
\text { stimulant, } \\
\text { gonorrhoea and } \\
\text { ulcer of bladder }\end{array}$ & $\begin{array}{l}\text { Bark, flower, } \\
\text { roots and } \\
\text { seeds }\end{array}$ & $\begin{array}{l}\text { Seed combine } \\
\text { with black } \\
\text { musli and } \\
\text { sufeed musli }\end{array}$ & $\begin{array}{l}1 \text { time per } \\
\text { day }\end{array}$ & $\begin{array}{l}\text { Dry } \\
\text { form }\end{array}$ & $\begin{array}{l}\text { Best for } \\
\text { diarrhoea, } \\
\text { dysentery }\end{array}$ \\
\hline $\begin{array}{l}\text { Brassica } \\
\text { campestris L. }\end{array}$ & Brassicaceae & Musturd, Saag & 0.46 & 0.04 & $\begin{array}{l}\text { Skin disorders, } \\
\text { cure of snake } \\
\text { bites }\end{array}$ & Flowers & $\begin{array}{l}\text { Oil got from } \\
\text { seeds of } \\
\text { mustard plant is } \\
\text { mix with leaf of } \\
\text { akk plant and } \\
\text { rubbed }\end{array}$ & $\begin{array}{l}\text { 3-4 times } \\
\text { per day }\end{array}$ & $\begin{array}{l}\text { Dry } \\
\text { form }\end{array}$ & Abdominal pain \\
\hline $\begin{array}{l}\text { Brassica } \\
\text { oleracea } \mathrm{L} .\end{array}$ & Brassicaceae & Cauliflower & 0.43 & 0.045 & $\begin{array}{l}\text { Laxative, } \\
\text { inflammation, } \\
\text { anticancer, } \\
\text { antioxidant } \\
\text { and neuro- } \\
\text { degenerative } \\
\text { disease }\end{array}$ & Leaves & $\begin{array}{l}\text { Take } 1 \text { cup } \\
\text { of chopped } \\
\text { cauliflower } \\
\text { leaves and } \\
\text { then cooked } \\
\text { properly }\end{array}$ & $\begin{array}{l}1 \text { time per } \\
\text { week }\end{array}$ & $\begin{array}{l}\text { Fresh } \\
\text { leaves }\end{array}$ & Control diabetes \\
\hline $\begin{array}{l}\text { Brassica } \\
\text { rapa L. }\end{array}$ & Brassicaceae & $\begin{array}{l}\text { Gonglu, } \\
\text { Shalgam, turnip }\end{array}$ & 0.46 & 0.055 & $\begin{array}{l}\text { Stomach } \\
\text { problem, } \\
\text { asthma, boosts } \\
\text { immune system, } \\
\text { atherosclerosis } \\
\text { and } \\
\text { cardiovascular } \\
\text { disease }\end{array}$ & $\begin{array}{l}\text { Leaves, fleshy } \\
\text { tap root, } \\
\text { seed }\end{array}$ & $\begin{array}{l}\text { Take leaves, } \\
\text { seed and then } \\
\text { ground it to } \\
\text { make powder. } \\
\text { This power is } \\
\text { recommended } \\
\text { for cancer } \\
\text { treatment }\end{array}$ & $\begin{array}{l}2 \text { times } \\
\text { per day }\end{array}$ & $\begin{array}{l}\text { Fresh } \\
\text { also dry } \\
\text { form }\end{array}$ & $\begin{array}{l}\text { Cancer } \\
\text { treatment }\end{array}$ \\
\hline $\begin{array}{l}\text { Brassica } \\
\text { campestris } \\
\text { var. sarson. } \\
\text { Prain }\end{array}$ & Brassicaceae & Toria & 0.56 & 0.07 & $\begin{array}{l}\text { Cold, influenza, } \\
\text { fever, bronchitis } \\
\text { and rheumatic } \\
\text { pains }\end{array}$ & Seed & $\begin{array}{l}\text { Oil obtained } \\
\text { from the seed } \\
\text { of toria plants } \\
\text { are used as } \\
\text { medicine to } \\
\text { treat number of } \\
\text { diseases }\end{array}$ & $\begin{array}{l}1 \text { time per } \\
\text { day }\end{array}$ & $\begin{array}{l}\text { Dry } \\
\text { form }\end{array}$ & $\begin{array}{l}\text { Cold, joints and } \\
\text { arthritis }\end{array}$ \\
\hline $\begin{array}{l}\text { Calotropis } \\
\text { procera (Ai- } \\
\text { ton) Dryand. }\end{array}$ & Apocyanaceae & $\begin{array}{l}\text { Akk, Sodom } \\
\text { apple, rubber } \\
\text { tree }\end{array}$ & 0.66 & 0.095 & $\begin{array}{l}\text { Deafness, } \\
\text { piles, baldness, } \\
\text { wound healing, } \\
\text { toothache, pain } \\
\text { reducing and } \\
\text { skin disease }\end{array}$ & $\begin{array}{l}\text { Leaves, seed, } \\
\text { flower root } \\
\text { and latex }\end{array}$ & $\begin{array}{l}\text { Oil got from } \\
\text { seeds of } \\
\text { mustard plant is } \\
\text { mix with leaf of } \\
\text { akk plant and } \\
\text { rubbed }\end{array}$ & $\begin{array}{l}\text { 3-4 times } \\
\text { per day }\end{array}$ & $\begin{array}{l}\text { Dry } \\
\text { from }\end{array}$ & $\begin{array}{l}\text { For any type of } \\
\text { body pain }\end{array}$ \\
\hline $\begin{array}{l}\text { Cannabis } \\
\text { sativa } \mathrm{L} .\end{array}$ & Cannabaceae & Bhaang & 0.8 & 0.075 & $\begin{array}{l}\text { Intoxication, } \\
\text { loss of appetite, } \\
\text { control } \\
\text { inflammation of } \\
\text { body, asthma, } \\
\text { bronchitis, } \\
\text { headache, flu, } \\
\text { cough and pains }\end{array}$ & Whole plant & $\begin{array}{l}\text { Seed and leaf } \\
\text { use to make } \\
\text { poultice }\end{array}$ & $\begin{array}{l}\text { Twice a } \\
\text { day }\end{array}$ & $\begin{array}{l}\text { Fresh } \\
\text { form }\end{array}$ & $\begin{array}{l}\text { For burn and } \\
\text { muscular pain }\end{array}$ \\
\hline $\begin{array}{l}\text { Carthamus } \\
\text { oxyacantha } \\
\text { M. Bieb. }\end{array}$ & Asteraceae & Poli & 0.5 & 0.058 & $\begin{array}{l}\text { Treat ulcer } \\
\text { problems, } \\
\text { stomach } \\
\text { infertility, } \\
\text { ulcer, problems } \\
\text { jaundice and also } \\
\text { cure scabies }\end{array}$ & Whole plant & $\begin{array}{l}\text { Oil is used as } \\
\text { tonic }\end{array}$ & $\begin{array}{l}1 \text { time } \\
\text { per day }\end{array}$ & $\begin{array}{l}\text { Dry } \\
\text { form }\end{array}$ & $\begin{array}{l}\text { Respiratory } \\
\text { problem }\end{array}$ \\
\hline $\begin{array}{l}\text { Carthamus } \\
\text { roseus } \mathrm{L} \text {. }\end{array}$ & Apocyanaceae & $\begin{array}{l}\text { Sada bahar, } \\
\text { Periwinkle }\end{array}$ & 0.53 & 0.095 & $\begin{array}{l}\text { Pain, headache, } \\
\text { immunity, } \\
\text { anticancer, } \\
\text { antidiabetics, } \\
\text { skin infection } \\
\text { and } \\
\text { cardiovascular } \\
\text { disease }\end{array}$ & $\begin{array}{l}\text { Root, shoot, } \\
\text { leaves }\end{array}$ & $\begin{array}{l}\text { Take sada bhar } \\
\text { leaves, boil } \\
\text { them and make } \\
\text { poultice }\end{array}$ & $\begin{array}{l}\text { Twice a } \\
\text { day }\end{array}$ & $\begin{array}{l}\text { Fresh } \\
\text { leaves }\end{array}$ & $\begin{array}{l}\text { Blood cancer, } \\
\text { diabetes, } \\
\text { Alzheimer }\end{array}$ \\
\hline $\begin{array}{l}\text { Cassia fistula } \\
\text { L. }\end{array}$ & Fabaceae & $\begin{array}{l}\text { Amaltas, } \\
\text { Golder tree }\end{array}$ & 0.66 & 0.065 & $\begin{array}{l}\text { Skin infection, } \\
\text { fever, ageusia, } \\
\text { constipation } \\
\text { purposes and for } \\
\text { pain reliving }\end{array}$ & $\begin{array}{l}\text { Bark, fruit, } \\
\text { leaves roots }\end{array}$ & $\begin{array}{l}5 \mathrm{~g} \text { fruit, } 2 \mathrm{~g} \\
\text { bark, } 1 \mathrm{~g} \text { leaves } \\
\text { and roots to } \\
\text { make decoction } \\
\text { use with water }\end{array}$ & $\begin{array}{l}\text { Twice a } \\
\text { day }\end{array}$ & $\begin{array}{l}\text { Fresh } \\
\text { form }\end{array}$ & Constipation \\
\hline
\end{tabular}




\begin{tabular}{|c|c|c|c|c|c|c|c|c|c|c|}
\hline $\begin{array}{l}\text { Chenopodium } \\
\text { album } \mathrm{L} \text {. }\end{array}$ & Amaranthaceae & Bathu & 0.7 & 0.08 & $\begin{array}{l}\text { Urinary } \\
\text { problems, skin } \\
\text { problems, } \\
\text { constipation, } \\
\text { jaundice and } \\
\text { laxative }\end{array}$ & $\begin{array}{l}\text { Leaves } \\
\text { and stem }\end{array}$ & $\begin{array}{l}3 \text { g leaves and } \\
\text { mix with water } \\
\text { and use for } \\
\text { kidney stone }\end{array}$ & $\begin{array}{l}\text { Twice a } \\
\text { day }\end{array}$ & $\begin{array}{l}\text { Dry } \\
\text { form }\end{array}$ & $\begin{array}{l}\text { Kidney store, } \\
\text { joint, skeleton }\end{array}$ \\
\hline $\begin{array}{l}\text { Cirsium } \\
\text { arvense (L.) } \\
\text { Scop. }\end{array}$ & Asteraceae & $\begin{array}{l}\text { California } \\
\text { thistle, Lay }\end{array}$ & 0.5 & 0.05 & $\begin{array}{l}\text { Toothache, tonic, } \\
\text { diuretic, liver } \\
\text { disorder and } \\
\text { treat worm in } \\
\text { children }\end{array}$ & Roots & & & & \\
\hline $\begin{array}{l}\text { Citrus limon } \\
\text { (L.) Osbeck }\end{array}$ & Rutaceae & Lemon & 0.43 & 0.025 & $\begin{array}{l}\text { Teeth infection, } \\
\text { stomach } \\
\text { problem }\end{array}$ & Fruits & $\begin{array}{l}\text { Take lemon } \\
\text { water } 6 \mathrm{~g} \text { and } \\
\text { add in herbal } \\
\text { tea }\end{array}$ & $\begin{array}{l}\text { Twice a } \\
\text { day }\end{array}$ & $\begin{array}{l}\text { Fresh } \\
\text { form }\end{array}$ & $\begin{array}{l}\text { For fever control } \\
\text { and hepatitis }\end{array}$ \\
\hline $\begin{array}{l}\text { Citrus para- } \\
\text { dise Macfad. }\end{array}$ & Rutaceae & Grape fruit & 0.4 & 0.07 & $\begin{array}{l}\text { Antiseptic, } \\
\text { astringent and } \\
\text { treat muscle } \\
\text { fatigue }\end{array}$ & $\begin{array}{l}\text { Whole plant } \\
\text { body }\end{array}$ & $\begin{array}{l}\text { Juice is use } \\
\text { for fever }\end{array}$ & $\begin{array}{l}\text { Twice a } \\
\text { day }\end{array}$ & Fresh & Fever control \\
\hline $\begin{array}{l}\text { Citrus sinensis } \\
\text { (L.) Osbeck }\end{array}$ & Rutaceae & Orange, Malta & 0.36 & 0.05 & $\begin{array}{l}\text { Fever, scurvy, } \\
\text { inflammation } \\
\text { and nervous } \\
\text { disorders }\end{array}$ & Flower & Juice of orange & $\begin{array}{l}\text { Thrice a } \\
\text { day }\end{array}$ & Fresh & $\begin{array}{l}\text { For liver and } \\
\text { kidney }\end{array}$ \\
\hline $\begin{array}{l}\text { Convolvulus } \\
\text { arvense L. }\end{array}$ & Convulvulaceae & $\begin{array}{l}\text { Lali, Vahri, } \\
\text { Bind Weed }\end{array}$ & 0.63 & 0.075 & $\begin{array}{l}\text { Constipation, } \\
\text { immunity, } \\
\text { anti-diabetics, } \\
\text { control anaemia, } \\
\text { menstrual } \\
\text { problem, } \\
\text { laxatives, fever } \\
\text { and also improve } \\
\text { poor vision }\end{array}$ & Whole plant & $\begin{array}{l}\text { Dry form of } \\
\text { leaves use } \\
\text { with milk }\end{array}$ & $\begin{array}{l}\text { Twice a } \\
\text { day }\end{array}$ & $\begin{array}{l}\text { Fresh } \\
\text { form }\end{array}$ & $\begin{array}{l}\text { For cough and } \\
\text { asthma, heart } \\
\text { problem }\end{array}$ \\
\hline $\begin{array}{l}\text { Coriandrum } \\
\text { sativum } \mathrm{L} .\end{array}$ & Apiaceae & Dania & 0.6 & 0.085 & $\begin{array}{l}\text { Stomach } \\
\text { problem, loss } \\
\text { of appetite, } \\
\text { diarrhoea, } \\
\text { joints problem, } \\
\text { toothache, } \\
\text { measles and } \\
\text { haemorrhoids }\end{array}$ & $\begin{array}{l}\text { Stems and } \\
\text { leaves }\end{array}$ & $\begin{array}{l}\text { Dania leaves } \\
\text { and seed }\end{array}$ & $\begin{array}{l}\text { Twice a } \\
\text { day }\end{array}$ & Fresh & $\begin{array}{l}\text { Increase memory } \\
\text { of human brain, } \\
\text { and cool up body }\end{array}$ \\
\hline $\begin{array}{l}\text { Cymbopogon } \\
\text { citratus (DC.) } \\
\text { Stapf }\end{array}$ & Poaceae & Lemon grass & 0.7 & 0.02 & $\begin{array}{l}\text { Stomach } \\
\text { problem, } \\
\text { obesity control, } \\
\text { headaches, } \\
\text { respiratory } \\
\text { problem, CNS } \\
\text { problem, fever, } \\
\text { insect repellent } \\
\text { and indigestion }\end{array}$ & Leaves & $\begin{array}{l}\text { Make herbal tea } \\
\text { from the leaves } \\
\text { of lemon grass } \\
\text { in which also } \\
\text { add sabaz ilachi } \\
\text { and dal chini }\end{array}$ & $\begin{array}{l}\text { Twice a } \\
\text { day }\end{array}$ & $\begin{array}{l}\text { Fresh } \\
\text { leaves }\end{array}$ & $\begin{array}{l}\text { Cholera and } \\
\text { stomach problem }\end{array}$ \\
\hline $\begin{array}{l}\text { Cynodon } \\
\text { dactylon (L.) } \\
\text { Pers. }\end{array}$ & Poaceae & $\begin{array}{l}\text { Bermudalbham } \\
\text { grass }\end{array}$ & 0.53 & 0.055 & $\begin{array}{l}\text { Laxative, } \\
\text { wounds } \\
\text { problem, } \\
\text { bronchial } \\
\text { disorders, and } \\
\text { also kidney } \\
\text { disorder }\end{array}$ & Whole plant & $\begin{array}{l}\text { Paste of bham } \\
\text { grass plant is } \\
\text { useful to cuts } \\
\text { and wounds }\end{array}$ & $\begin{array}{l}2-3 \text { times } \\
\text { per day }\end{array}$ & $\begin{array}{l}\text { Fresh } \\
\text { part }\end{array}$ & $\begin{array}{l}\text { Peripheral } \\
\text { bleeding }\end{array}$ \\
\hline $\begin{array}{l}\text { Dactylis } \\
\text { glomerata L. }\end{array}$ & Poaceae & Barnyard grass & 0.43 & 0.035 & $\begin{array}{l}\text { Fever, tumours, } \\
\text { kidney and } \\
\text { bladder ailments }\end{array}$ & $\begin{array}{l}\text { Flower and } \\
\text { stem }\end{array}$ & $\begin{array}{l}\text { Take leaves } \\
\text { fresh and dry }\end{array}$ & $\begin{array}{l}1 \text { time } \\
\text { per day }\end{array}$ & Fresh & Fever \\
\hline $\begin{array}{l}\text { Dalbergia } \\
\text { sissoo DC. }\end{array}$ & Fabaceae & Tali, Shisham & 0.7 & 0.075 & $\begin{array}{l}\text { Nose bleed, } \\
\text { gonorrhoea, stop } \\
\text { vomiting, boils } \\
\text { and skin disorder }\end{array}$ & Leaves & $\begin{array}{l}\text { Take } 2 \mathrm{~g} \text { leaves } \\
\text { and fruit }\end{array}$ & $\begin{array}{l}\text { Twice a } \\
\text { day }\end{array}$ & $\begin{array}{l}\text { Fresh } \\
\text { form }\end{array}$ & Epilepsy \\
\hline $\begin{array}{l}\text { Eclipta } \\
\text { prostrata } \\
\text { (L.) L. }\end{array}$ & Asteraceae & $\begin{array}{l}\text { White twin } \\
\text { head }\end{array}$ & 0.56 & 0.52 & $\begin{array}{l}\text { Allergy, } \\
\text { athlete's foot } \\
\text { and ringworm, } \\
\text { constipation, } \\
\text { fever, body pain, } \\
\text { bp problem }\end{array}$ & Leaves & $\begin{array}{l}\text { Plant juice } \\
\text { mix with } \\
\text { essential oil }\end{array}$ & $\begin{array}{l}1 \text { time } \\
\text { per day }\end{array}$ & $\begin{array}{l}\text { Fresh } \\
\text { form }\end{array}$ & Use for jaundice \\
\hline
\end{tabular}




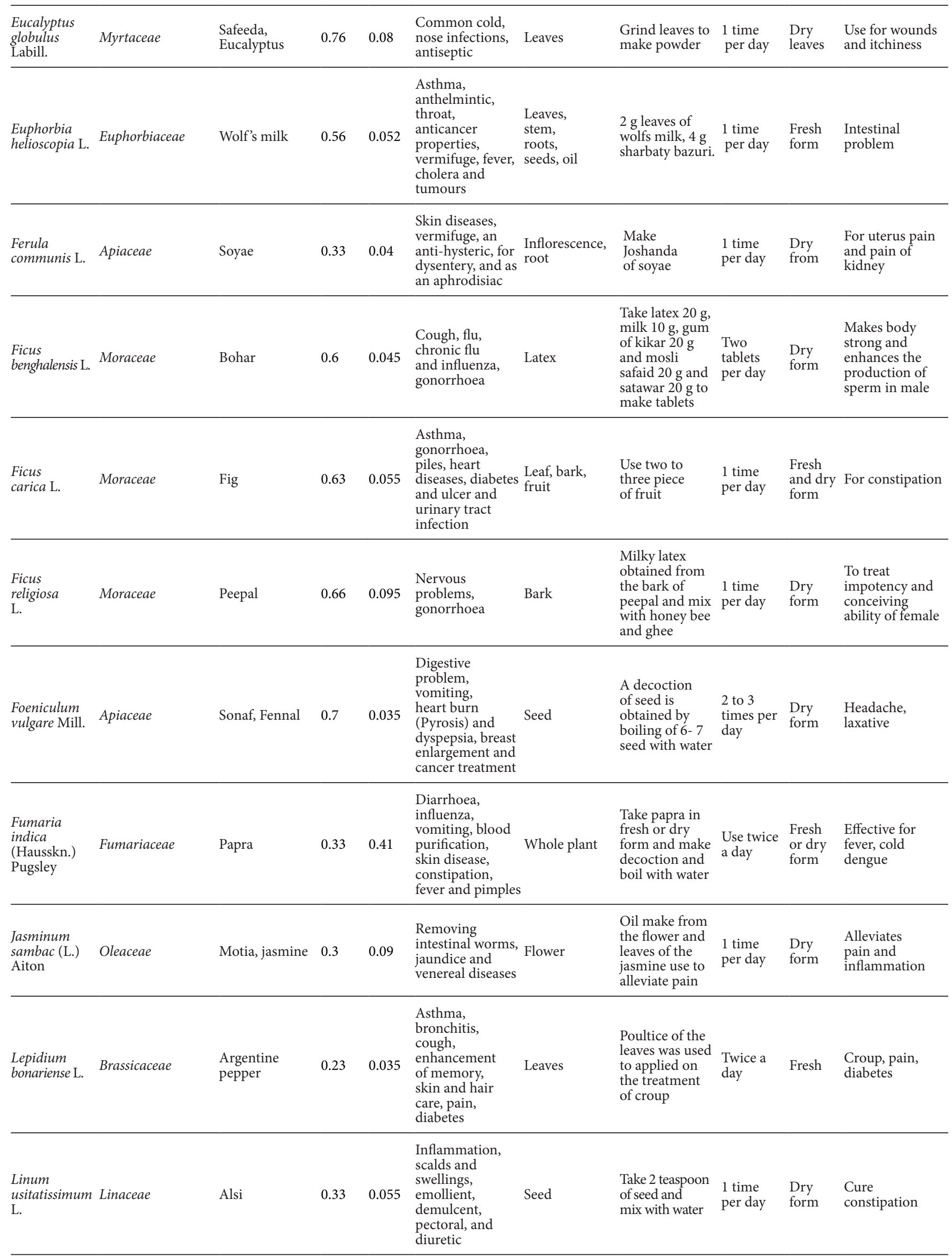




\begin{tabular}{|c|c|c|c|c|c|c|c|c|c|c|}
\hline $\begin{array}{l}\text { Lycopus } \\
\text { exaltatus L.f. }\end{array}$ & Lamiaceae & Cut leaves & 0.5 & 0.065 & $\begin{array}{l}\text { Skin problem, } \\
\text { astringent, } \\
\text { sedative and also } \\
\text { for tuberculosis, } \\
\text { narcotic, } \\
\text { contraceptive } \\
\text { effect }\end{array}$ & $\begin{array}{l}\text { Flower, stem } \\
\text { and leaves }\end{array}$ & $\begin{array}{l}\text { Leaves of this } \\
\text { plant applied } \\
\text { directly to } \\
\text { healing of } \\
\text { wounds }\end{array}$ & $\begin{array}{l}2-3 \text { times } \\
\text { per day }\end{array}$ & Fresh & $\begin{array}{l}\text { Healing of } \\
\text { wound }\end{array}$ \\
\hline $\begin{array}{l}\text { Malva } \\
\text { sylvestris L. }\end{array}$ & Malvaceae & $\begin{array}{l}\text { Blue mallow, } \\
\text { Pick-cheese }\end{array}$ & 0.33 & 0.095 & $\begin{array}{l}\text { Wound } \\
\text { healing, ulcers } \\
\text { and bladder, } \\
\text { indigestion, } \\
\text { gastric mucus, } \\
\text { relaxing activity } \\
\text { and diuretic }\end{array}$ & Leaves, flower & $\begin{array}{l}\text { Take leaves and } \\
\text { flower of this } \\
\text { plant and put } \\
\text { into warmly } \\
\text { water for few } \\
\text { hour }\end{array}$ & $\begin{array}{l}2-3 \text { times } \\
\text { per day }\end{array}$ & $\begin{array}{l}\text { Fresh } \\
\text { form }\end{array}$ & Cough cure \\
\hline $\begin{array}{l}\text { Mangifera } \\
\text { indica L. }\end{array}$ & Anacardiaceae & Amm, Mango & 0.4 & 0.085 & $\begin{array}{l}\text { Improper } \\
\text { digestion, ear } \\
\text { pain, vomiting } \\
\text { and stomach } \\
\text { disorder }\end{array}$ & Leaf, seed & $\begin{array}{l}1 \text { seed pulp of } \\
\text { mango and } 1 \\
\text { teaspoon of } \\
\text { corn flour } \\
\text { mixed with it }\end{array}$ & $\begin{array}{l}\text { Take one } \\
\text { time in } \\
\text { morning } \\
\text { in a day }\end{array}$ & $\begin{array}{l}\text { Fresh } \\
\text { form }\end{array}$ & $\begin{array}{l}\text { To control } \\
\text { diabetes }\end{array}$ \\
\hline $\begin{array}{l}\text { Medicago } \\
\text { sativa } \mathrm{L} \text {. }\end{array}$ & Fabaceae & $\begin{array}{l}\text { Barseen, } \\
\text { Shatala, alfalfa }\end{array}$ & 0.43 & 0.045 & $\begin{array}{l}\text { High cholesterol } \\
\text { level and asthma, } \\
\text { osteoarthritis, } \\
\text { diabetes, upset } \\
\text { stomach, } \\
\text { bleeding disorder }\end{array}$ & $\begin{array}{l}\text { Flower and } \\
\text { seed }\end{array}$ & $\begin{array}{l}\text { Take } 10 \text { g each } \\
\text { as ginsing, oat, } \\
\text { alfalfa and } \\
\text { use to make } \\
\text { syrup }\end{array}$ & $\begin{array}{l}\text { Adult t } \\
\text { ake twice } \\
\text { a day }\end{array}$ & Fresh & $\begin{array}{l}\text { To control } \\
\text { mental fatigue, } \\
\text { loss of appetite }\end{array}$ \\
\hline $\begin{array}{l}\text { Melia } \\
\text { azedarach L. }\end{array}$ & Meliaceae & Dareek & 0.46 & 0.065 & $\begin{array}{l}\text { Ulcer and skin } \\
\text { problems, gout, } \\
\text { inflammation }\end{array}$ & $\begin{array}{l}\text { Leaves and } \\
\text { root }\end{array}$ & $\begin{array}{l}\text { Leaves of } \\
\text { dareek, Gandak } \\
\text { amla saar, jamal } \\
\text { gota of equal } \\
\text { volume to } \\
\text { prepare tablets }\end{array}$ & $\begin{array}{l}4 \text { times } \\
\text { per day } \\
\text { with milk }\end{array}$ & $\begin{array}{l}\text { Dry } \\
\text { form }\end{array}$ & Ulcer, itchiness \\
\hline $\begin{array}{l}\text { Mentha } \\
\text { arvensis } \mathrm{L} .\end{array}$ & Lamiaceae & Podina, Mint & 0.63 & 0.06 & $\begin{array}{l}\text { Cough, } \\
\text { mouthwash, } \\
\text { herbal tea and } \\
\text { digestive } \\
\text { problem }\end{array}$ & $\begin{array}{l}\text { Leaves and } \\
\text { stem }\end{array}$ & $\begin{array}{l}\text { Take podina } \\
\text { leaves and } \\
\text { ajwain, ginger } \\
\text { and noshadar } \\
10 \mathrm{~g} \text { and black } \\
\text { paper } 10 \mathrm{~g} \text { to } \\
\text { make decoction }\end{array}$ & $\begin{array}{l}\text { Trice } \\
\text { a day }\end{array}$ & $\begin{array}{l}\text { Dry } \\
\text { form }\end{array}$ & $\begin{array}{l}\text { For digestive } \\
\text { purpose }\end{array}$ \\
\hline $\begin{array}{l}\text { Morus } \\
\text { nigra L. }\end{array}$ & Moraceae & Shatoot & 0.6 & 0.09 & $\begin{array}{l}\text { Colds, eye } \\
\text { infections and } \\
\text { nose bleeds, } \\
\text { asthma, } \\
\text { influenza, } \\
\text { cough, bronchitis } \\
\text { and diabetes }\end{array}$ & $\begin{array}{l}\text { Bark and } \\
\text { leaves }\end{array}$ & $\begin{array}{l}\text { Shatoot fruit } \\
\text { and malathi }\end{array}$ & $\begin{array}{l}\text { Trice } \\
\text { a day }\end{array}$ & $\begin{array}{l}\text { Fresh } \\
\text { form }\end{array}$ & $\begin{array}{l}\text { Sore of throat } \\
\text { and for cough }\end{array}$ \\
\hline $\begin{array}{l}\text { Oxalis } \\
\text { corniculata L. }\end{array}$ & Oxalidaceae & $\begin{array}{l}\text { Miyan, } \\
\text { Creeping } \\
\text { wood-sorrel }\end{array}$ & 0.63 & 0.045 & $\begin{array}{l}\text { Dysentery, } \\
\text { digestive } \\
\text { problems, } \\
\text { diuretic, } \\
\text { diarrhea, } \\
\text { urinary tract } \\
\text { infections, skin } \\
\text { rashes, digestive } \\
\text { problems, fever } \\
\text { and influenza }\end{array}$ & Leaves & $\begin{array}{l}\text { Dry leaves } \\
\text { grind to make } \\
\text { powder }\end{array}$ & $\begin{array}{l}2 \text { times } \\
\text { per day }\end{array}$ & $\begin{array}{l}\text { Dry } \\
\text { form }\end{array}$ & $\begin{array}{l}\text { Use for snake } \\
\text { bite }\end{array}$ \\
\hline $\begin{array}{l}\text { Parthenium } \\
\text { hysterophorus } \\
\text { L. }\end{array}$ & Asteraceae & $\begin{array}{l}\text { Santa maria, } \\
\text { Gandi booti }\end{array}$ & 0.36 & 0.07 & $\begin{array}{l}\text { Treat toothache, } \\
\text { kill tape worm, } \\
\text { cure malaria }\end{array}$ & $\begin{array}{l}\text { Leaves and } \\
\text { flower }\end{array}$ & $\begin{array}{l}\text { Decoction of } \\
\text { gandi booti is } \\
\text { use in } \\
\text { traditional } \\
\text { medicine }\end{array}$ & $\begin{array}{l}1 \text { times } \\
\text { per day }\end{array}$ & $\begin{array}{l}\text { Fresh } \\
\text { form }\end{array}$ & $\begin{array}{l}\text { Fever, urinary } \\
\text { track infection }\end{array}$ \\
\hline $\begin{array}{l}\text { Pedalium } \\
\text { murex L. }\end{array}$ & Pedaliaceae & $\begin{array}{l}\text { Pakhra, } \\
\text { Gokhru }\end{array}$ & 0.26 & 0.055 & $\begin{array}{l}\text { Cough, cold, } \\
\text { antiseptic and } \\
\text { gonorrhoea }\end{array}$ & Leaves & $\begin{array}{l}\text { Take leaves of } \\
\text { pakhra and } \\
\text { seed of two } \\
\text { gram grind to } \\
\text { make powder }\end{array}$ & $\begin{array}{l}2 \text { times } \\
\text { per day }\end{array}$ & $\begin{array}{l}\text { Dry or } \\
\text { fresh } \\
\text { form }\end{array}$ & $\begin{array}{l}\text { Purification of } \\
\text { blood }\end{array}$ \\
\hline $\begin{array}{l}\text { Pennisetum } \\
\text { mezianum } \\
\text { Leeke }\end{array}$ & Poaceae & $\begin{array}{l}\text { Lynda, Barner } \\
\text { grass }\end{array}$ & 0.33 & 0.055 & $\begin{array}{l}\text { Appetizer, } \\
\text { tonic, heart } \\
\text { diseases } \\
\text { and pimples, } \\
\text { cataracts and } \\
\text { wound healing }\end{array}$ & $\begin{array}{l}\text { Leaves and } \\
\text { stem }\end{array}$ & $\begin{array}{l}\text { Plant leave use } \\
\text { to make } \\
\text { powder and } \\
\text { mix with honey }\end{array}$ & $\begin{array}{l}3 \text { times } \\
\text { per day }\end{array}$ & $\begin{array}{l}\text { Fresh } \\
\text { form }\end{array}$ & For cough \\
\hline
\end{tabular}




\begin{tabular}{|c|c|c|c|c|c|c|c|c|c|c|}
\hline $\begin{array}{l}\text { Phoenix } \\
\text { dactylifera L. }\end{array}$ & Arecaceae & Date, Khajur & 0.7 & 0.075 & $\begin{array}{l}\text { Fever, diarrhea, } \\
\text { general body } \\
\text { weakness, } \\
\text { respiratory } \\
\text { diseases and also } \\
\text { help to reduce } \\
\text { blood pressure }\end{array}$ & Fruits & $\begin{array}{l}\text { Kajor, badam, } \\
\text { pista, } \\
\text { karamphal } \\
\text { and sonaf are } \\
\text { homogenize }\end{array}$ & $\begin{array}{l}2 \text { times } \\
\text { per day } \\
\text { with milk }\end{array}$ & $\begin{array}{l}\text { Take } \\
\text { dry } \\
\text { sample }\end{array}$ & $\begin{array}{l}\text { Control general } \\
\text { weakness }\end{array}$ \\
\hline $\begin{array}{l}\text { Psidium } \\
\text { guajava L. }\end{array}$ & Myrtaceae & Amrood, Guava & 0.73 & 0.175 & $\begin{array}{l}\text { Cancerous } \\
\text { disease and also } \\
\text { improve appetite, } \\
\text { cure stomach } \\
\text { prooblem }\end{array}$ & Fruit & $\begin{array}{l}\text { Recent research } \\
\text { observed that } \\
\text { eating of large } \\
\text { amount of } \\
\text { guava daily up } \\
\text { to } 12 \text { weeks its } \\
\text { lowering the } \\
\text { blood pressure }\end{array}$ & $\begin{array}{l}3-4 \text { times } \\
\text { per day }\end{array}$ & Fresh & $\begin{array}{l}\text { Control high } \\
\text { blood pressure }\end{array}$ \\
\hline $\begin{array}{l}\text { Punica } \\
\text { granatum L. }\end{array}$ & Lythraceae & $\begin{array}{l}\text { Anaar, pome } \\
\text { granate }\end{array}$ & 0.63 & 0.058 & $\begin{array}{l}\text { Stomach } \\
\text { disorders, } \\
\text { menstural } \\
\text { irregularities }\end{array}$ & Fruit, bark & $\begin{array}{l}12 \mathrm{~g} \text { Japanese } \\
\text { fruit, } 12 \mathrm{~g} \text { bark } \\
\text { of anaar, } 12 \mathrm{~g} \\
\text { kutraj are mix } \\
\text { with } 11 \text { water } \\
\text { to make } 250 \mathrm{~g} \\
\text { solution }\end{array}$ & $\begin{array}{l}3 \text { times } \\
\text { per day }\end{array}$ & $\begin{array}{l}\text { Fresh } \\
\text { form }\end{array}$ & $\begin{array}{l}\text { Intestinal } \\
\text { infection }\end{array}$ \\
\hline $\begin{array}{l}\text { Raphanus } \\
\text { sativus L. }\end{array}$ & Brassicaceae & Mooli, radish & 0.33 & 0.05 & $\begin{array}{l}\text { Liver and } \\
\text { stomach } \\
\text { problem, } \\
\text { weight loss, } \\
\text { antidiabetics, } \\
\text { intestinal } \\
\text { disease and for } \\
\text { renal problem }\end{array}$ & $\begin{array}{l}\text { Underground } \\
\text { part }\end{array}$ & $\begin{array}{l}\text { Take moli } \\
\text { leaves and } \\
\text { sugar grind } \\
\text { it to get sap }\end{array}$ & $\begin{array}{l}1 \text { time } \\
\text { per day }\end{array}$ & $\begin{array}{l}\text { Fresh } \\
\text { form }\end{array}$ & Hepatitis \\
\hline $\begin{array}{l}\text { Ricinus } \\
\text { communis L. }\end{array}$ & Euphorbiaceae & $\begin{array}{l}\text { Andol, Castor } \\
\text { oil }\end{array}$ & 0.6 & 0.055 & $\begin{array}{l}\text { Bowels } \\
\text { problems, } \\
\text { constipation } \\
\text { and stomach } \\
\text { problem }\end{array}$ & Seed & $\begin{array}{l}6 \mathrm{~g} \text { root } \\
\text { and milk }\end{array}$ & $\begin{array}{l}1 \text { time } \\
\text { per day }\end{array}$ & $\begin{array}{l}\text { Dry } \\
\text { form }\end{array}$ & $\begin{array}{l}\text { Liver and } \\
\text { hepatitis }\end{array}$ \\
\hline Rosa indica $\mathrm{L}$. & Rosaceae & Rose, Ghulaab & 0.73 & 0.075 & $\begin{array}{l}\text { Heart disorders, } \\
\text { eye infection, } \\
\text { asthma, break } \\
\text { stone from } \\
\text { kidney and } \\
\text { gall bladder }\end{array}$ & $\begin{array}{l}\text { Seed } \\
\text { and flower }\end{array}$ & $\begin{array}{l}\text { Gulgand } 1 \mathrm{~g} \text {, } \\
\text { badam } 50 \mathrm{~g} \\
\text { and sagmonia } \\
10 \mathrm{~g}\end{array}$ & $\begin{array}{l}\text { Only } \\
\text { night } \\
\text { time }\end{array}$ & $\begin{array}{l}\text { Dry } \\
\text { form }\end{array}$ & $\begin{array}{l}\text { For constipation } \\
\text { and appetizer }\end{array}$ \\
\hline $\begin{array}{l}\text { Saccharum } \\
\text { officinarium L. }\end{array}$ & Poaceae & Shorgum, Gana & 0.7 & 0.045 & $\begin{array}{l}\text { Skin, urinary } \\
\text { tract infections, } \\
\text { syrup, jaundice, } \\
\text { hemorrhage, } \\
\text { baked product } \\
\text { and other urinary } \\
\text { disease }\end{array}$ & Stem & $\begin{array}{l}\text { Unripe fruit of } \\
\text { grapefruit and } \\
\text { gana juice is } \\
\text { mixed up }\end{array}$ & $\begin{array}{l}\text { One cup } \\
\text { per day } \\
\text { for }\end{array}$ & $\begin{array}{l}\text { Fresh } \\
\text { sample }\end{array}$ & Dysentery \\
\hline $\begin{array}{l}\text { Saccharum } \\
\text { spontaneum } \mathrm{L} .\end{array}$ & Poaceae & $\begin{array}{l}\text { European } \\
\text { beach grass, } \\
\text { saroot }\end{array}$ & 0.4 & 0.04 & $\begin{array}{l}\text { Appetite, } \\
\text { antimicrobial, } \\
\text { antibacterial and } \\
\text { antifungal. }\end{array}$ & Whole plant & $\begin{array}{l}\text { Leave grinder } \\
\text { to make } \\
\text { joshanda }\end{array}$ & $\begin{array}{l}\text { Twice a } \\
\text { day }\end{array}$ & & For constipation \\
\hline $\begin{array}{l}\text { Solanum } \\
\text { lycopersicum } \\
\mathrm{L} .\end{array}$ & Solanaceae & Tomato & 0.33 & 0.05 & $\begin{array}{l}\text { Skin disease, } \\
\text { toothache }\end{array}$ & $\begin{array}{l}\text { Fruit } \\
\text { and leaves }\end{array}$ & $\begin{array}{l}\text { Gridded } \\
\text { tomato }\end{array}$ & $\begin{array}{l}1 \text { time } \\
\text { per day }\end{array}$ & Fresh & Produce RBCs \\
\hline $\begin{array}{l}\text { Spinacia } \\
\text { oleracea } \mathrm{L} .\end{array}$ & Amaranthaceae & Palak & 0.23 & 0.04 & $\begin{array}{l}\text { Anti-diabetic, } \\
\text { anti-bacterial and } \\
\text { liver treatment, } \\
\text { blood builder, } \\
\text { promoting } \\
\text { growth in } \\
\text { children and } \\
\text { appetite stimulant }\end{array}$ & Leaves & Salan of palak & $\begin{array}{l}2 \text { times } \\
\text { per day }\end{array}$ & Fresh & $\begin{array}{l}\text { Inflammation of } \\
\text { stomach }\end{array}$ \\
\hline $\begin{array}{l}\text { Stellaria } \\
\text { media (L.) } \\
\text { Vill. }\end{array}$ & Caryophyllaceae & $\begin{array}{l}\text { Common } \\
\text { chickweed }\end{array}$ & 0.36 & 0.025 & $\begin{array}{l}\text { Cough, kidney } \\
\text { irregularities, } \\
\text { treat itchy skin } \\
\text { conditions and } \\
\text { pulmonary } \\
\text { diseases }\end{array}$ & $\begin{array}{l}\text { Leaves and } \\
\text { flowers }\end{array}$ & $\begin{array}{l}\text { Take chickweed } \\
\text { leaves and } \\
\text { applied directly } \\
\text { to the surface } \\
\text { of skin }\end{array}$ & $\begin{array}{l}2 \text { times } \\
\text { per day }\end{array}$ & $\begin{array}{l}\text { Fresh } \\
\text { leaves }\end{array}$ & $\begin{array}{l}\text { Cure skin } \\
\text { problem and } \\
\text { ulcer }\end{array}$ \\
\hline $\begin{array}{l}\text { Syzygium } \\
\text { cumini (L.) } \\
\text { Skeels }\end{array}$ & Myrtaceae & Jamanu & 0.5 & 0.08 & $\begin{array}{l}\text { Sore throat, } \\
\text { diabetics and } \\
\text { ulcers }\end{array}$ & $\begin{array}{l}\text { Seeds, fruit } \\
\text { barks, leaves }\end{array}$ & $\begin{array}{l}1 \text { to } 2 \mathrm{~g} \text { powder } \\
\text { of jaman seed } \\
\text { mixed with } 1 \mathrm{~g} \\
\text { water for one } \\
\text { night. Helpful } \\
\text { for high BP }\end{array}$ & $\begin{array}{l}\text { Use in } \\
\text { the } \\
\text { morning } \\
\text { for } 15 \text { days }\end{array}$ & $\begin{array}{l}\text { Dry } \\
\text { form }\end{array}$ & Diabetes \\
\hline
\end{tabular}




\begin{tabular}{|c|c|c|c|c|c|c|c|c|c|c|}
\hline $\begin{array}{l}\text { Tagetes erecta } \\
\text { L. }\end{array}$ & Asteraceae & Marigold, gutta & 0.43 & 0.045 & $\begin{array}{l}\text { Treat } \\
\text { indigestion, } \\
\text { pyrexia, } \\
\text { earache, } \\
\text { intestinal } \\
\text { parasites, } \\
\text { muscular pain, } \\
\text { antifungal and } \\
\text { cure piles }\end{array}$ & $\begin{array}{l}\text { Flower and } \\
\text { leaves }\end{array}$ & $\begin{array}{l}\text { Paste of } \\
\text { marigold } \\
\text { use for cut } \\
\text { of wound }\end{array}$ & $\begin{array}{l}2 \text { times } \\
\text { per day }\end{array}$ & $\begin{array}{l}\text { Fresh } \\
\text { leaves }\end{array}$ & $\begin{array}{l}\text { External } \\
\text { bleeding }\end{array}$ \\
\hline $\begin{array}{l}\text { Triticum } \\
\text { aestivum } \mathrm{L} .\end{array}$ & Poaceae & $\begin{array}{l}\text { Wheat, } \\
\text { gandum }\end{array}$ & 0.26 & 0.06 & $\begin{array}{l}\text { Constipation, } \\
\text { control colon } \\
\text { diseases, } \\
\text { reducing risk } \\
\text { of cancer and } \\
\text { gallbladder }\end{array}$ & Seeds & $\begin{array}{l}\text { Take seed of } \\
\text { gandum and } \\
\text { then heat up } \\
\text { in the oil. } \\
\text { Obtained sab } \\
\text { is rubbed on } \\
\text { the wounds }\end{array}$ & $\begin{array}{l}2 \text { to } 3 \\
\text { times } \\
\text { per day }\end{array}$ & $\begin{array}{l}\text { Dry } \\
\text { form }\end{array}$ & Cure wounds \\
\hline $\begin{array}{l}\text { Vitis vinifera } \\
\text { L. }\end{array}$ & Vitaceae & Angoor, grapes & 0.33 & 0.065 & $\begin{array}{l}\text { Fever, vomiting, } \\
\text { weight loss and } \\
\text { heart diseases }\end{array}$ & Fruit & $\begin{array}{l}2 \mathrm{~g} \text { leaves } \\
\text { and } 1 \mathrm{~g} \text { water } \\
\text { to make one } \\
\text { cup and then } \\
\text { mix fruit of } \\
\text { angoor }\end{array}$ & $\begin{array}{l}\text { Use twice } \\
\text { a day }\end{array}$ & Fresh & Hematemesis \\
\hline $\begin{array}{l}\text { Withania } \\
\text { somnifera (L.) } \\
\text { Dunal }\end{array}$ & Solanaceae & $\begin{array}{l}\text { Aksan, } \\
\text { Ashwaghandha }\end{array}$ & 0.63 & 0.035 & $\begin{array}{l}\text { Asthma, spasm, } \\
\text { rheumatism, } \\
\text { artheroschlerois } \\
\text { and sedation }\end{array}$ & $\begin{array}{l}\text { Leaves and } \\
\text { flowers }\end{array}$ & $\begin{array}{l}\text { Take root of } \\
\text { aksan about } \\
300 \mathrm{~g} \text { daily } \\
\text { after food }\end{array}$ & $\begin{array}{l}\text { Twice a } \\
\text { day }\end{array}$ & Fresh & $\begin{array}{l}\text { Improves } \\
\text { symptoms of } \\
\text { stress }\end{array}$ \\
\hline Zea mays L. & Poaceae & Makai, maize & 0.66 & 0.095 & $\begin{array}{l}\text { Bladder, urinary } \\
\text { disorders and } \\
\text { kidney disorders }\end{array}$ & $\begin{array}{l}\text { Stigma of } \\
\text { female } \\
\text { flower }\end{array}$ & $\begin{array}{l}\text { Makai is used } \\
\text { as flour }\end{array}$ & $\begin{array}{l}\text { Thrice a } \\
\text { day }\end{array}$ & Dry & $\begin{array}{l}\text { Weakness } \\
\text { overcome, } \\
\text { vomiting }\end{array}$ \\
\hline $\begin{array}{l}\text { Ziziphus } \\
\text { jujuba Mill. }\end{array}$ & Rhamnaceae & Beri & 0.63 & 0.057 & $\begin{array}{l}\text { Skin infection } \\
\text { and control } \\
\text { deficiency } \\
\text { of iron }\end{array}$ & $\begin{array}{l}\text { Leaves and } \\
\text { fruits }\end{array}$ & $\begin{array}{l}\text { Use fruit of } \\
\text { beri plant in } \\
\text { present form }\end{array}$ & $\begin{array}{l}\text { Twice a } \\
\text { day }\end{array}$ & $\begin{array}{l}\text { Dry } \\
\text { form }\end{array}$ & $\begin{array}{l}\text { Hepatitis, } \\
\text { stomach disorder }\end{array}$ \\
\hline
\end{tabular}

(16\%), seed (16\%), bark (14\%), root (13\%), whole plant (12\%), underground part (3\%) and latex 3\% (fig. 3).

\section{Informant consensus factor (ICF) and fidelity level (FL)}

According to this study it was noted that ICF value ranged from 0.27 to 0.67 (tab. 2) with the lowest values for gonorrhoea and diabetes (0.27), and the highest values were noted in stomach and skin infection (0.67). Fidelity level was used to find out the floral species for pharmaceutical purposes. The FL value ranged from 21 to 91. Magnifera indica had the highest FL of 91, whereas Allium sativum had the lowest, at 21. Collected ethnomedicinal flora had numerous curative stuffs (tab. 3). Achyranthes aspera was used mostly to treat cough,

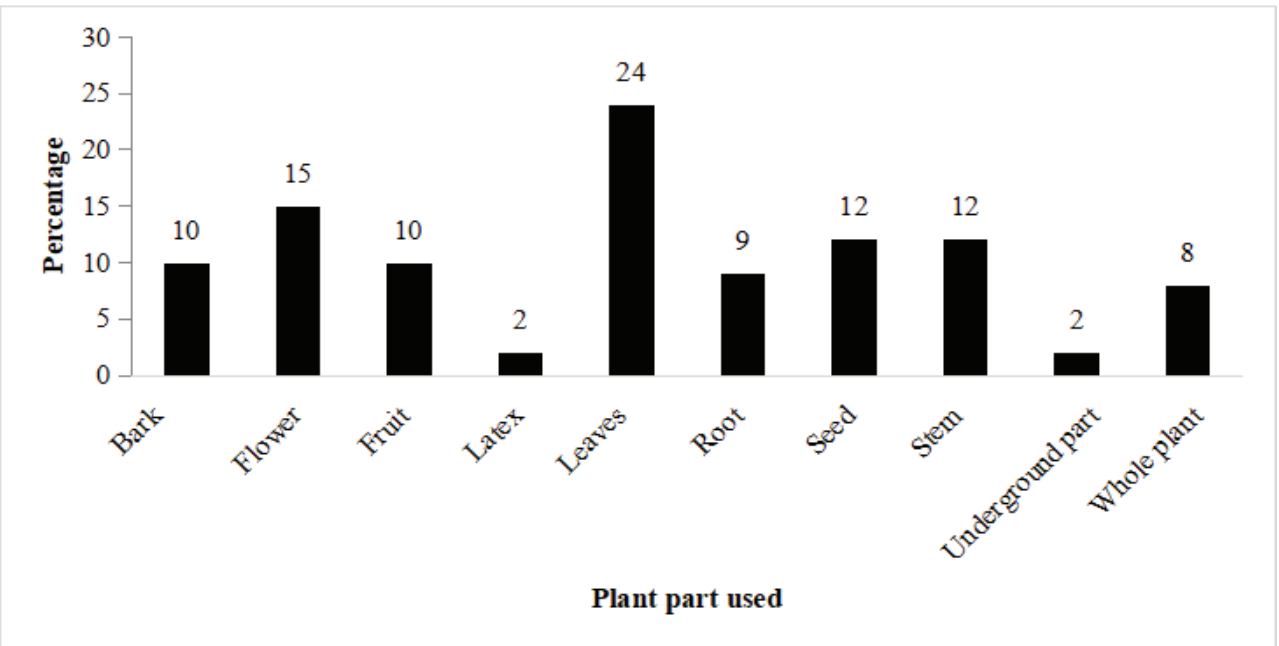

Figure 3

Family index with highest number of species used in study area 
kidney problems, dysentery, skin disease, rheumatism, stomach pain and toothache. Mangifera indica was recommended to treat improper digestion, ear pain, vomiting and stomach disorder. Phoenix dactylifera was used to cure fever, diarrhoea, general body weakness, respiratory diseases and to reduce blood pressure. Ricinus communis was medicated in bowel, constipation and stomach diseases. According to respondent, these ethnomedicinal flora was also suggested in the treatment of different types of other diseases including: cardiovascular disorders, respiratory diseases, hepatitis, gastric problems, kidney, urogenital problems, liver disorders, constipation, diarrhoea, cold, fever, cough, asthma, bronchitis, flu, intestinal problems, vomiting, headache, arthritis, hypertension, paralysis, malaria, cancer, allergies, tumor, male infertility, piles, sexual disorders, snake bite, rheumatism, toothache, stomach problems, insect bite, body pain, nervous disorders infections, diabetes, swellings, eye problems, pneumonia, bone pain, jaundice and joint swellings.

It was observed that Achyranthes aspera had the lowest fidelity level (FL) of $63 \%$ and was used for kidney problems, cough, dysentery, rheumatism, skin disease, stomach pain and toothache but mainly recommended to get relief from cough using its leaves and honey thrice a day. Calotropis procera had $68 \% \mathrm{FL}$ and was used to cure piles, skin diseases, deafness and pain reducing. Majority of people consume the leaves of this plant mixed with mustard oil in wound healing and body pain for 3 to 4 times a day. The FL calculated for Chenopodium album and Psidium guajava was $69 \%$ which plays a vital role in the treatment of various health disorders, i.e. jaundice, laxative and urinary problems and improving appetite. Phoenix dactylifera exhibited $75 \%$ FL. It was widely used for reducing blood pressure, fever and respiratory ailments. Syzygium cumini showed $81 \%$ FL, and was used for various purposes such as ulcer and sore throat. Cannabis sativa exhibiting FL 84\%, was used for the control of cough, pains, asthma and bad appetite. It was noted from the data that Cynodon dactylon had $86 \%$ FL and was utilized to cure kidney, wound and respiratory disorders. The fidelity level of Mangifera indica was $91 \%$ and it was used to cure stomach disorders, vomiting and improper digestion. Rosa indica had the highest FL of $95 \%$ and was utilized in kidney, gall bladder and heart diseases (tab. 3).

\section{DISCUSSION}

This study has shown that district of Gujrat is rich in a variety of plants which are used by native people to cure many diseases. They also can be used in pharmaceutical industry. Local residents rely on floral species for the traditional ingesting. Consequently, bulk of labours termed the diversity of species in diverse zone and scrutinizes their pharmaceutical uses. Mahmood et al. [11] designated that Withania somnifera was recycled to treat spasms, sexual disorder, sedate patients and rheumatic. Amaranthus viridis was very useful in curing eye disorders, urinary

Table 2

Informant Consensus Factor (ICF) by disease category in the study area

\begin{tabular}{|c|c|c|c|c|c|}
\hline Disease category & $\begin{array}{l}\text { No. of use reports } \\
\text { (Nur) }\end{array}$ & $\begin{array}{l}\text { No. of species used } \\
\text { (Nt) }\end{array}$ & Nur-Nt & Nur-1 & ICF \\
\hline Flu, fever, cough, cold & 22 & 13 & 9 & 21 & 0.42 \\
\hline Heart, kidney, urinary, ulcer and liver problem & 26 & 15 & 11 & 25 & 0.44 \\
\hline Gonorrhoea, diabetes, diarrhoea & 19 & 12 & 5 & 18 & 0.27 \\
\hline Nose, ear, eye and teeth problems & 13 & 5 & 8 & 12 & 0.66 \\
\hline $\begin{array}{l}\text { Asthma, rheumatism, lungs, bronchitis, } \\
\text { jaundice, blood problems }\end{array}$ & 21 & 13 & 8 & 20 & 0.4 \\
\hline $\begin{array}{l}\text { Appetizer, constipation, inflammation, hair } \\
\text { problem }\end{array}$ & 17 & 7 & 10 & 16 & 0.62 \\
\hline $\begin{array}{l}\text { Stomach problems, skin problems, sexual } \\
\text { problems }\end{array}$ & 35 & 12 & 23 & 34 & 0.67 \\
\hline Piles, arthritis, atherosclerosis, female problems & 10 & 5 & 5 & 9 & 0.55 \\
\hline
\end{tabular}


Table 3

Fidelity Level (FL) value of commonly reported medicinal plants

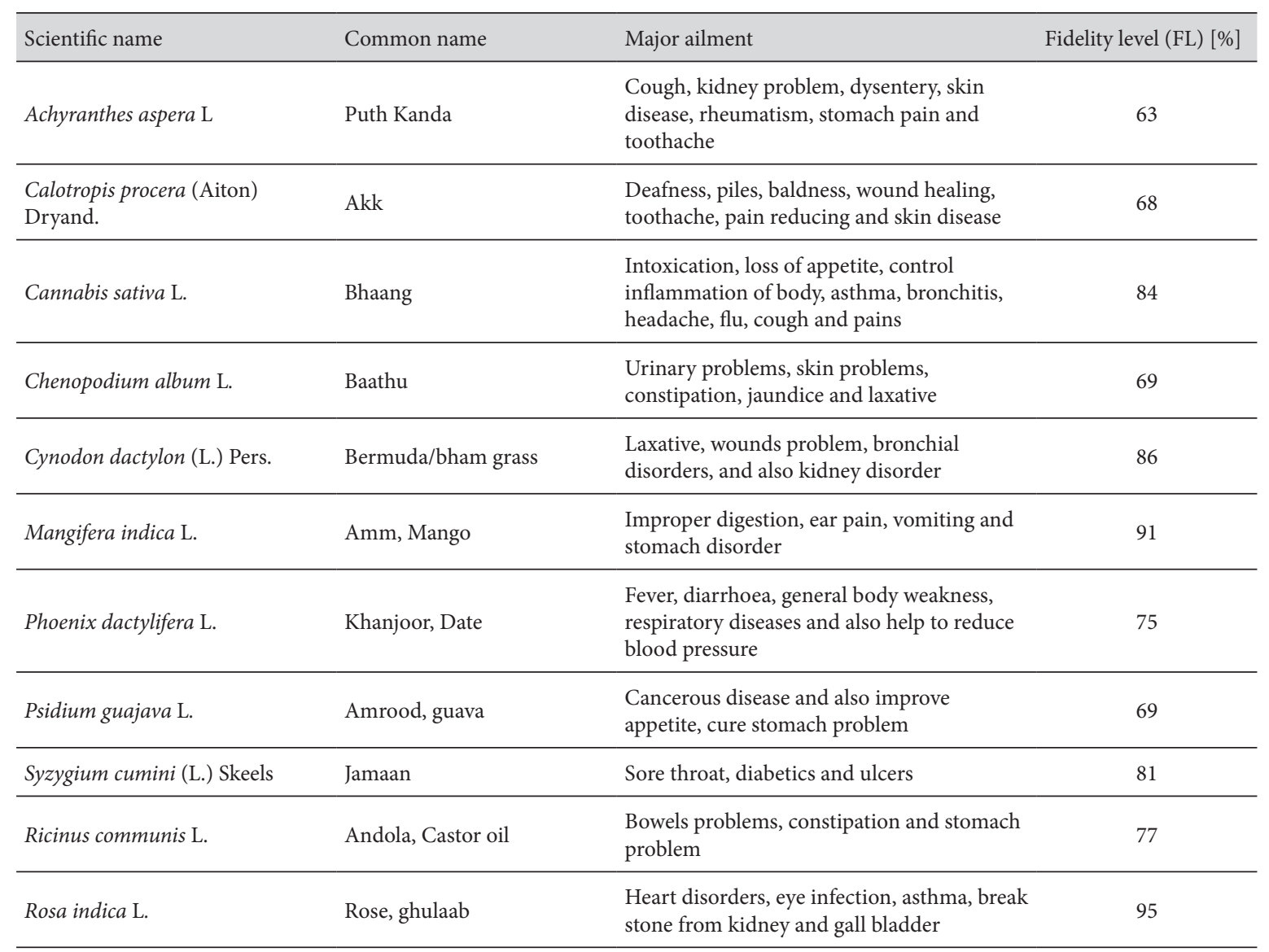

disorders and menstrual problems [12]. Mangifera indica was useful for vomiting, ear pain and stomach disorders [13]. Calotropis procera was used for baldness, piles, deafness, wound healing, reduction of pain, toothache and skin diseases [14].

During ethnomedicinal study, it was observed that Cynodon dactylon was used to treat all types of bleeding, laxative, bronchial disorders, wounds problem, kidney disorder and also skin troubles [15]. Bombax ceiba was used against inflammation, snake bites, blood purification, dysentery, as a stimulant, in gonorrhoea, genital organs treatment and ulcer of bladder [14]. Avena sativa was useful in the treatment of tension, constipation and skin allergies [16].

Albuquerque et al. [17] conducted a study to show that the most common therapeutic prpoerties were performed by plants with the values (UV) as follows: Psidium guajava (2.52), Cannabis sativa (0.98) and of Cordyline fruticose (0.03). The highest fidelity level displayed the prominence of specific floral species by instinctive persons to treat explicit disease. The high FL value presented the use of a specific ethnomedicinal plants by the indigenous people to treat a complex disease [18]. Amiri and Joharchi [9] testified that Tagetes erecta was valuable to cure earache, indigestion, pyrexia, intestinal washes, intestinal parasites, antifungal, muscular pain and cure piles. Syzygium cumini was used as a remedy of sore throat, diabetes and ulcers [19]. Euphorbia helioscopia was used for handling of breathing disorders including asthma, anthelmintic, bronchitis, chest congestion, throat, vermifuge. It has also anticancer properties, cures cholera, fever and tumours [20]. The study of all these ethnomedicinal plants showed the noteworthy value of innate inhabitants of the community. It shows that medicinal plants must be activated rather than to be left as surplus. This flora can be vended to several herbal industry for the preparation of voluminous brands that are also favourable to get money [2]. It has also been described that Gujrat district had many plants that are considered as weeds but are important medicinal plants of the area used by local community [1]. 


\section{CONCLUSIONS}

It was concluded that there were many useful plants in this area. These plants are of great significance among the local community and are used to cure many diseases.

\section{ACKNOWLEDGMENTS}

Authors would like to pay thank for faculty and staff of the Department of Botany, University of Gujrat, Pakistan.

Conflict of interest: The authors declare no conflict of interest.

\section{REFERENCES}

1. Hussain K, Nisar MF, Majeed A, Nawaz K. Ethnomedicinal survey for important plants of Jalalpur Jattan, district Gujrat, Punjab, Pakistan. Ethnobot Leaflets 2010;20:11. https://opensiuc.lib.siu.edu/cgi/ viewcontent.cgi?article $=1670 \&$ context $=\mathrm{ebl}$

2. Hussain K, Shahazad A, Zia-ul-Hussnain S. An ethnobotanical survey of important wild medicinal plants of Hattar district Haripur, Pakistan. Ethnobot Leaflets 2008; 5:29-35. https://opensiuc.lib.siu.edu/cgi/viewcontent. cgi? article $=1039 \&$ context $=\mathrm{ebl}$

3. Shinwari ZK. Ethnobotany in Pakistan: sustainable and participatory approach. In: Proc. 1st Training Workshop on Ethnobotany and its Application to Conservation NARC. J Biol Environ Sci 1996; 10:14-25. https://academicjournals.org/ article/article1380378394_Shinwari.pdf

4. Qureshi RA, Ghufran MA, Gilani SA. Ethnobotanical studies of selected medicinal plants of Sudhan gali and Ganga chotti hills, district Bagh, Azad Kashmir. Pak J Bot 2007; 39:2275-2283. https://pdfs.semanticscholar.org/74f2/3187d5c24 0bce878ce234ccb28a10b4f2376.pdf

5. Shah A, Marwat SK, Gohar F. Ethnobotanical study of medicinal plants of semi-tribal area of Makerwal and Gulla Khel (lying between Khyber Pakhtunkhwa and Punjab Provinces), Pakistan. Amer J Plant Sci 2013; 4:98. http://dx.doi. org.10.4236/ajps.2013.41015
6. Hameed M, Ashraf M, Al-Quriany F. Medicinal flora of the Cholistan desert: a review. Pak J Bot 2011; 43:39-50. http://www.pakbs.org/pjbot/ PDFs/43(SI)/07.pdf

7. Govindappa M, Poojashri MN. Antimicrobial, antioxidant and in vitro anti-inflammatory activity of ethanol extract and active phytochemical screening of Wedelia trilobata (L.) Hitchc. J Pharma Phytother 2011; 3:43-51. http://www. academicjournals.org/jpp

8. Khan MA, Mujtaba G, Hussain M. Ethnobotanical study about medicinal plants of Poonch valley Azad Kashmir. J Animal Plant Sci 2012; 22:493500. http://thejaps.org.pk/docs/v-22-2/42.pdf

9. Amiri MS, Joharchi MR. 2013. Ethnobotanical investigation of traditional medicinal plants commercialized in the markets of Mashhad, Iran. Avicenna J Phytomed 2013; 3:254. https://www. ncbi.nlm.nih.gov/pmc/articles/PMC4075713/ pdf/ajp-3-254.pdf

10. Arshad M, Ahmad M, Ahmed E. An ethnobiological study in Kala Chitta hills of Pothwar region, Pakistan: multinomial logit specification. J Ethnobio Ethnomed 2014; 10:13. http://www. ethnobiomed.com/content/10/1/13

11. Mahmood A, Mujtaba G. Indigenous medicinal knowledge of common plants from district Kotli Azad Jammu and Kashmir Pakistan. J Med Plants Res 2012; 6:4961-4967. doi: http://dx.doi. org/10.5897/JMPR12.703

12. Iqbal MJ, Hanif $S$, Mahmoo $Z$ et al. Antioxidant and antimicrobial activities of Chowlai (Amaranthus viridis L.) leaf and seed extracts. J Med Plants Res 2012; 6: 4450-4455. doi: http://dx.doi. org/10.5897/JMPR12.822

13. Shah KA, Patel MB, Patel RJ. Mangifera indica (mango). Pharmacognosy Rev 2010; 4:42. doi: http://dx.doi.org.10.4103/0973-7847.65325

14. Harne S, Sharma A, Dhaygude M. et al. Novel route for rapid biosynthesis of copper nanoparticles using aqueous extract of Calotropis procera L. latex and their cytotoxicity on tumor cells. Colloids Surf B Biointerfaces J 2012; 95:284-288. doi: http://dx.doi.org.10.1016/j.colsurfb.2012.03.005

15. Singh SK. Ethno-medicinal plants of Kullu valley, 
Himachal Pradesh. J Non-Timber Forest Prod 2004; 11:74-79. https://interscience.org.uk/v3i5/1\%20ijahm.pdf

16. Ajaib M, Ashraf Z, Riaz F. Ethnobotanical studies of some plants of tehsil Kharian, district Gujrat. FUUAST J Bio 2014; 4:65-71. http://fuuastjb.org/ index.php/fuuastjb/article/view/220

17. Albuquerque UP, Lucena RF, Monteiro JM. Evaluating two quantitative ethnobotanical techniques. Ethnobot Res Appl 2006; 4:51-60. http:// www.ethnobotanyjournal.org/index.php/era/article/view/101/87

18. Ugulu I, Baslar S, Yorek N. The investigation and quantitative ethnobotanical evaluation of me- dicinal plants used around Izmir province, Turkey. J Med Plant Res 2009; 3:345-367. https:// academicjournals.org/journal/JMPR/article-abstract/BE1B7DF15170

19. Qureshi R, Maqsood M, Arshad M. Ethnomedicinal uses of plants by the people of Kadhi areas of Khushab, Punjab, Pakistan. Pak J Bot 2011; 43:121-133. http://www.pakbs.org/pjbot/ PDFs/43\%281\%29/PJB43\%281\%29121.pdf

20. Kumar P, Yadava RK, Gollen B. Nutritional contents and medicinal properties of wheat: a review. Life Sci Med Res 2011; 22:1-10. https://astonjournals.com/manuscripts/Vol2011/LSMR-22_ Vol2011.pdf 